\title{
Vulvar dermatoses: a histopathologic review and classification of 183 cases $\dagger$
}

Background: Vulvar dermatoses are often difficult to classify due to histopathologic overlap. We aimed to report our experience at a single institution.

Methods: A total of 183 non-neoplastic, non-infectious vulvar biopsies were reviewed. Associations between histopathologic features and specific diagnoses were analyzed by Chi-squared tests. Results: Twenty-two biopsies (12.0\%) showed two concurrent processes. A limited differential rather than a definitive diagnosis was rendered in 15 cases $(8.2 \%)$. The final diagnoses included lichen sclerosus (LS) $(38.8 \%)$, lichen simplex chronicus (LSC) $(29.0 \%)$, eczematous dermatitis $(23.0 \%)$, Zoon vulvitis $(8.2 \%)$, non-specific/resolved dermatitis $(5.5 \%)$, hidradenitis suppurativa $(2.7 \%)$, Behçet disease $(2.2 \%)$, lichen planus (1.6\%), ruptured cyst $(1.6 \%)$, ulcer not-otherwise-specified $(1.6 \%)$, psoriasis $(1.1 \%)$, radiation dermatitis $(1.1 \%)$, sebopsoriasis $(1.1 \%)$, seborrheic dermatitis $(1.1 \%)$, epidermolytic hyperkeratosis $(0.5 \%)$ and granular parakeratosis $(0.5 \%)$. Early LS and Zoon vulvitis were commonly included as part of a differential diagnosis. LS was associated with wiry collagen with lymphocyte entrapment $(\mathrm{p}=0.0188)$. LSC was associated with zones of pale epithelium $(\mathrm{p}=0.0084)$, and often displayed prominent fibroblasts $(\mathrm{p}=0.0555)$. Zoon vulvitis was frequently misdiagnosed, and was associated with basal keratinocytic crowding $(\mathrm{p}<0.0001)$.

Conclusions: Our study has determined the relative frequencies of a wide variety of vulvar dermatoses, and identified new diagnostic clues for early LS, LSC and Zoon vulvitis.

Keywords: lichen sclerosus, lichen simplex chronicus, vulva, Zoon vulvitis

Chan MP, Zimarowski MJ. Vulvar dermatoses: a histopathologic review and classification of 183 cases $\dagger$.

J Cutan Pathol 2015; 42: 510-518. (C) 2015 John Wiley \& Sons A/S. Published by John Wiley \& Sons Ltd

\section{May P. Chan ${ }^{1}$ and Mary Jane Zimarowski $^{2}$}

${ }^{1}$ Department of Pathology and Department of Dermatology, University of Michigan, Ann Arbor, MI, USA and

${ }^{2}$ Department of Pathology, Beth Israel

Deaconess Medical Center, Harvard Medical School, Boston, MA, USA

$\uparrow$ Presented at the 101st Annual Meeting of the United States and Canadian Academy of Pathology, Vancouver, BC, Canada, 17-23 March 2012.
May P. Chan, MD,

Department of Pathology and Department of Dermatology, University of Michigan, 1301 Catherine Street, Medical Science I, M3261, Ann Arbor, Ml 48109-5602, USA

Tel: +7347644460

Fax: +7347644690

e-mail:mpchan@med.umich.edu

Accepted for publication November 25, 2014
While well-developed vulvar dermatoses such as late-stage lichen sclerosus (LS) may be easy to recognize both clinically and pathologically, vulvar biopsies taken for early LS or other inflammatory conditions are often difficult to classify. Owing to the unique location and anatomy of the vulva, understanding of vulvar dermatoses requires knowledge in both dermatopathology and gynecologic pathology. In many practices, however, dermatopathologists 
Table 1. The 2006 International Society for the Study of Vulvovaginal Disease (ISSVD) classification of non-neoplastic and non-infectious vulvar diseases ${ }^{1,2}$

\begin{tabular}{lc}
\hline Histologic patterns & Clinical correlates \\
\hline Spongiotic & $\begin{array}{c}\text { Atopic dermatitis, allergic contact } \\
\text { dermatitis, irritant contact dermatitis } \\
\text { Psoriasis, lichen simplex chronicus }\end{array}$ \\
$\begin{array}{l}\text { Acanthotic } \\
\text { Lichenoid } \\
\text { Dermal homogenization/ } \\
\text { sclerosis }\end{array}$ & $\begin{array}{c}\text { Lichen sclerosus, lichen planus } \\
\text { Vesiculobullous }\end{array}$ \\
& Cicatricial pemphigoid, linear IgA \\
Acantholytic & disease \\
& Hailey-Hailey disease, Darier disease, \\
Granulomatous & papular genitocrural acantholysis \\
& Crohn disease, Melkersson-Rosenthal \\
Vasculopathic & syndrome \\
& Aphthous ulcer, Behçet disease, Zoon \\
& vulvitis \\
\hline
\end{tabular}

have limited exposure to vulvar specimens as these lesions are infrequently biopsied by dermatologists. On the other hand, gynecologic pathologists may not be versed in the complexities of dermatologic disorders, especially with early and subtle cases where significant pathologic overlaps exist or changes may be minimal. As a result, only few pathologists are specialized in this field, and vulvar dermatoses remain susceptible to inadequate diagnosis and suboptimal treatment.

The International Society for the Study of Vulvovaginal Disease (ISSVD) has developed a classification system for vulvar diseases based on microscopic patterns in an attempt to standardize terminology used by multidisciplinary physicians. ${ }^{1,2}$ On the basis of this classification system, the pathologist may report a vulvar biopsy by its primary histopathologic pattern when a definitive diagnosis cannot be attained, and the receiving clinician may interpret the result by referring to its possible clinical correlates (Table 1). While this general scheme provides an excellent basis for clinicopathologic correlation, pathologists should still make every attempt to provide a precise diagnosis whenever possible. It is also important to realize that this broad classification does not address more subtle pathologic features and has omitted a number of less common conditions such as radiation dermatitis, seborrheic dermatitis, hidradenitis suppurativa, fixed drug eruption and graft-versus-host disease.

To date, only few studies in the English literature have reviewed the relative frequencies of biopsy-proven vulvar dermatoses. ${ }^{3-5}$ Most recognized LS and/or lichen simplex chronicus (LSC) as the most common diagnoses after exclusion of neoplastic and infectious cases. Other common dermatoses included eczema, psoriasis and lichen planus (LP). Most of these studies were limited by the lack of more refined classification. There was also a tendency to group less common conditions into a 'non-specific' or 'others' category. The lack of consistency in terminology, such as 'hypertrophic vulvitis', 'neurodermatitis', ${ }^{6}$ 'erosive vulvitis', ${ }^{4}$ and 'dermatitis'" without further specification in the older literature also created confusion.

The objective of our study is to document our experience in classifying vulvar dermatoses encountered at a single institution, including the relative frequencies of these conditions and the identification of subtle histopathologic features that are useful in narrowing the differential diagnoses. Our ultimate goal is to facilitate more accurate pathologic diagnoses that would help guide effective treatment in patients suffering from vulvar dermatoses.

\section{Materials and methods}

The surgical pathology archive at Beth Israel Deaconess Medical Center was searched for vulvar biopsies received between 2006 and 2010 . Neoplastic and infectious cases were excluded based on the original diagnoses on the pathology reports. The original glass slides of the remaining cases were retrieved, and the corresponding clinical data, when available, were obtained from electronic medical records. The cases were then reviewed by the investigators independently while blinded to the original diagnoses.

The histopathologic features examined and recorded for each case are listed in Table 2. Some of the features are defined as follows. Zones of pale epithelium refers to distinct clusters of pale keratinocytes within the epidermis/epithelium not attributable to glycogenation. Basal keratinocytic crowding refers to increased density of small and uniform basal keratinocytes. Wiry fibrosis with lymphocyte entrapment refers to abnormal thickened collagen within the superficial dermis/subepithelial stroma resulting in a single-filing or branching arrangement of the entrapped lymphocytes. Lastly, prominent fibroblasts refers to enlarged and/or multinucleated stromal cells with stellate cytoplasm.

The final diagnoses were determined based on consensus among the investigators' and the original pathologist's impressions, as well as clinical correlation wherever available. Relative 
Chan \& Zimarowski

Table 2. Specific histopathologic features recorded in this study

\begin{tabular}{ll}
\hline Epidermis/epithelium & Dermis/stroma \\
\hline Parakeratosis & Edema and/or sclerosis \\
Orthokeratosis & Band-like infiltrate \\
Acanthosis & Wiry fibrosis with \\
Atrophy & Prominocyte entrapment \\
Ulceration/erosion & Hemorrhage/hemosiderin \\
Zones of pale epithelium & Eosinophils \\
Basal keratinocytic crowding & Neutrophils \\
Basal vacuolar degeneration & Lymphocytes \\
Cytoid/colloid bodies & Plasma cells \\
Eosinophils & \\
Neutrophils &
\end{tabular}

frequency of each entity was calculated. Associations between specific histopathologic features and the final diagnoses were analyzed by Chi-squared tests. Statistical significance is defined by a p-value of less than 0.05 .

\section{Results}

A total of 188 vulvar biopsies were retrieved from the pathology archive. Five of these cases were subsequently excluded upon review due to the identification of Corynebacterium minutissimum (four cases) and herpesvirus (one case) not reported in the original diagnoses (Fig. 1). The remaining 183 cases, including specimens received from both gynecology and dermatology clinics, were analyzed. The average age of the patients at the time of biopsy was 53.6 years. Clinical data were available in 170 cases $(92.9 \%)$, which included brief descriptions of the symptoms (e.g. 'pruritus' and 'vulvar discomfort') and/or clinical signs (e.g. 'erythema' and 'white lesion'). Upon microscopic examination, 22 cases $(12.0 \%)$ showed evidence of two concurrent processes in a single biopsy, most (18 cases) of which involved superimposed LSC. A primary process was identified in 18 of 53 cases $(34.0 \%)$ of LSC, which included eczematous dermatitis (12 cases) and LS (6 cases). In 17 cases $(9.3 \%)$ a limited differential rather than a definitive diagnosis was rendered due to significant overlapping features and lack of clinical correlation. Ten cases $(5.5 \%)$ showed minimal or non-specific changes that were non-diagnostic or suggestive of resolving dermatitis ('non-specific/resolved dermatitis'). Another three cases (1.6\%) demonstrated ulcer and acute inflammation which could not be further classified ['ulcer not-otherwise-specified (NOS)'].
A
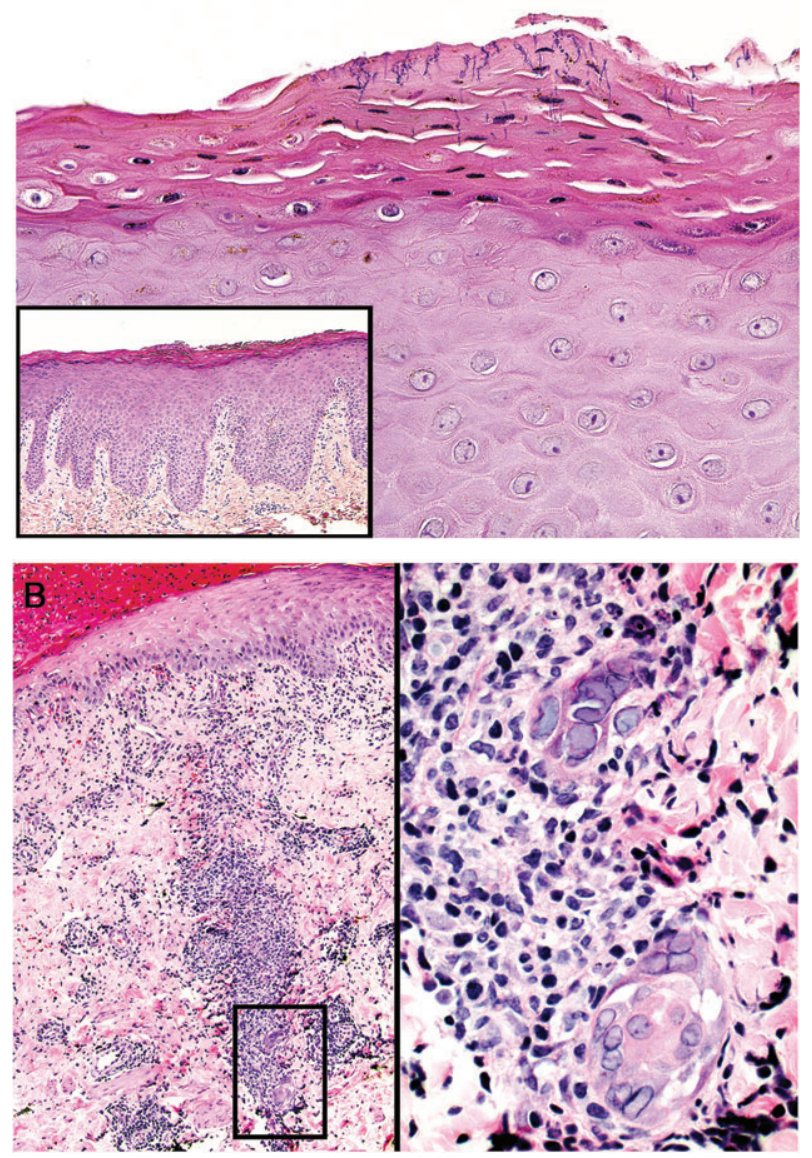

Fig. 1. Infections originally misdiagnosed. A) Erythrasma characterized by mild parakeratosis at scanning magnification (inset), where multiple vertically oriented bacterial bacilli are found upon close inspection. B) Herpesvirus infection characterized by perivascular inflammation and overlying inflamed scale crust at low magnification (left). Higher magnification of the adnexal structures (selected area, left) shows pathognomonic intranuclear viral inclusions affecting eccrine epithelial cells (right). [Hematoxylin-eosin, original magnifications $\times 600(\mathrm{~A}), \times 100(\mathrm{~B}$, left $)$, and $\times 600(\mathrm{~B}$, right $)]$.

All conditions encountered are listed in Table 3. Cases of LS were subdivided into early, mid and late stages based on the degree of lichenoid infiltrate (early) and well-developed stromal sclerosis (late). Cases of eczematous dermatitis were characterized by spongiosis, and were subdivided based on presumed etiologies: irritant contact dermatitis (ICD) as suggested by the presence of necrotic keratinocytes and/or intraepidermal neutrophils, and allergic contact dermatitis (ACD) as suggested by eosinophilic spongiosis. The remaining cases were classified as 'eczema NOS'.

The conditions most commonly included as part of a differential diagnosis were early LS (seven cases) and Zoon vulvitis (six cases). The 
Table 3. Final diagnoses of 183 cases in descending order of frequency

\begin{tabular}{lc}
\hline Final diagnoses & Number of cases $(\%)^{*}$ \\
\hline Lichen sclerosus & $71(38.8)$ \\
$\quad$ Early & $23(12.6)$ \\
Mid & $33(18.0)$ \\
Late & $15(8.2)$ \\
Lichen simplex chronicus & $53(29.0)$ \\
Eczematous (spongiotic) dermatitis & $42(23.0)$ \\
Allergic contact dermatitis & $13(7.1)$ \\
Irritant contact dermatitis & $9(4.9)$ \\
Eczema NOS & $20(10.9)$ \\
Zoon vulvitis & $15(8.2)$ \\
Non-specific/resolved dermatitis & $10(5.5)$ \\
Hidradenitis suppurativa & $5(2.7)$ \\
Behçet disease & $4(2.2)$ \\
Lichen planus & $3(1.6)$ \\
Ruptured cyst & $3(1.6)$ \\
Ulcer NOS & $3(1.6)$ \\
Psoriasis & $2(1.1)$ \\
Radiation dermatitis & $2(1.1)$ \\
Sebopsoriasis & $2(1.1)$ \\
Seborrheic dermatitis & $2(1.1)$ \\
Epidermolytic hyperkeratosis & $1(0.5)$ \\
Granular parakeratosis & $1(0.5)$ \\
\hline
\end{tabular}

NOS, not otherwise specified.

*Total percentage exceeds $100 \%$ because of multiple diagnoses in some cases.

differential diagnosis of early LS included Zoon vulvitis (three cases), eczematous dermatitis (two cases) and LP (two cases). The differential diagnosis of Zoon vulvitis included early LS (three cases), eczematous dermatitis (two cases) and Behçet disease (one case). Eight out of 15 cases $(53.3 \%)$ of Zoon vulvitis were originally diagnosed as LP, early LS, eczematous dermatitis (ICD) and psoriasis.

Wiry fibrosis with lymphocyte entrapment (Fig. 2A) was observed in $53.5 \%$ of the LS cases, and the association was statistically significant $(p=0.0188)$. In comparison, this feature was only seen in $17.0 \%$ of LSC and $14.3 \%$ of eczematous dermatitis not associated with LS. Prominent fibroblasts (Fig. 2B) was most commonly seen in LSC (54.7\% of cases), but the association fell slightly short of statistical significance $(p=0.0555)$. On the other hand, zones of pale epithelium (Fig. 2C) showed significant association with LSC $(p=0.0084)$, and was observed in $75.5 \%$ of these cases. Basal keratinocytic crowding (Fig. 2D) was observed in the vast majority $(93.3 \%)$ of the Zoon vulvitis cases $(p<0.0001)$.

No significant difference was observed in the proportion of cases containing eosinophils among different vulvar dermatoses (intraepidermal eosinophils, $\mathrm{p}=0.6324$; dermal eosinophils, $\mathrm{p}=0.7755)$. Eosinophils, usually sparse, were found in 35 of 71 cases $(49.3 \%)$ of LS; of these, 29 cases showed only dermal eosinophils, 5 cases showed both intraepidermal and dermal eosinophils and 1 case showed only intraepidermal eosinophils.

\section{Discussion}

Clinical distinction between neoplastic and inflammatory diseases of the vulva can be challenging, let alone the distinction among different vulvar dermatoses. A low threshold for biopsy is desirable not only to exclude neoplastic conditions but also to allow for early diagnosis and timely treatment of inflammatory diseases. The pathologist's role in making the correct diagnoses on vulvar biopsies is therefore critical. In addition to mucocutaneous disorders specific to the anogenital region, the vulvar skin is also subject to dermatologic disorders not restricted to the genitalia. For this reason, pathologists who sign out vulvar biopsies should be familiar with both genital and extragenital dermatoses, and keep a broad differential diagnosis in mind when interpreting these cases.

In this study, we sought to provide a comprehensive list of vulvar dermatoses encountered at an academic medical center, and the relative frequencies of these conditions. Our data show that the finding of two concurrent processes in a single biopsy is quite common, and most frequently involves LSC superimposed on another condition. This is not surprising as many vulvar dermatoses, such as LS and eczema, are pruritic and prone to chronic rubbing. In almost one tenth of the cases a definitive diagnosis could not be attained, and a limited differential diagnosis was rendered. This is in part attributable to inadequate clinical data available, but also reflects the diagnostic difficulty due to significant overlapping histopathologic features. A small number of cases showed minimal histopathologic changes insufficient for any specific diagnosis. It is possible that these patients may have undergone treatment prior to biopsy, although the limited clinical data available for these cases preclude definitive conclusion.

Our data reveal LS to be the most common vulvar dermatosis. However, such high frequency may be in part due to the lower threshold for biopsy in patients with an established history of LS, given its increased risk of developing differentiated vulvar intraepithelial neoplasia and squamous cell carcinoma. ${ }^{7-11}$ The 


\section{Chan \& Zimarowski}
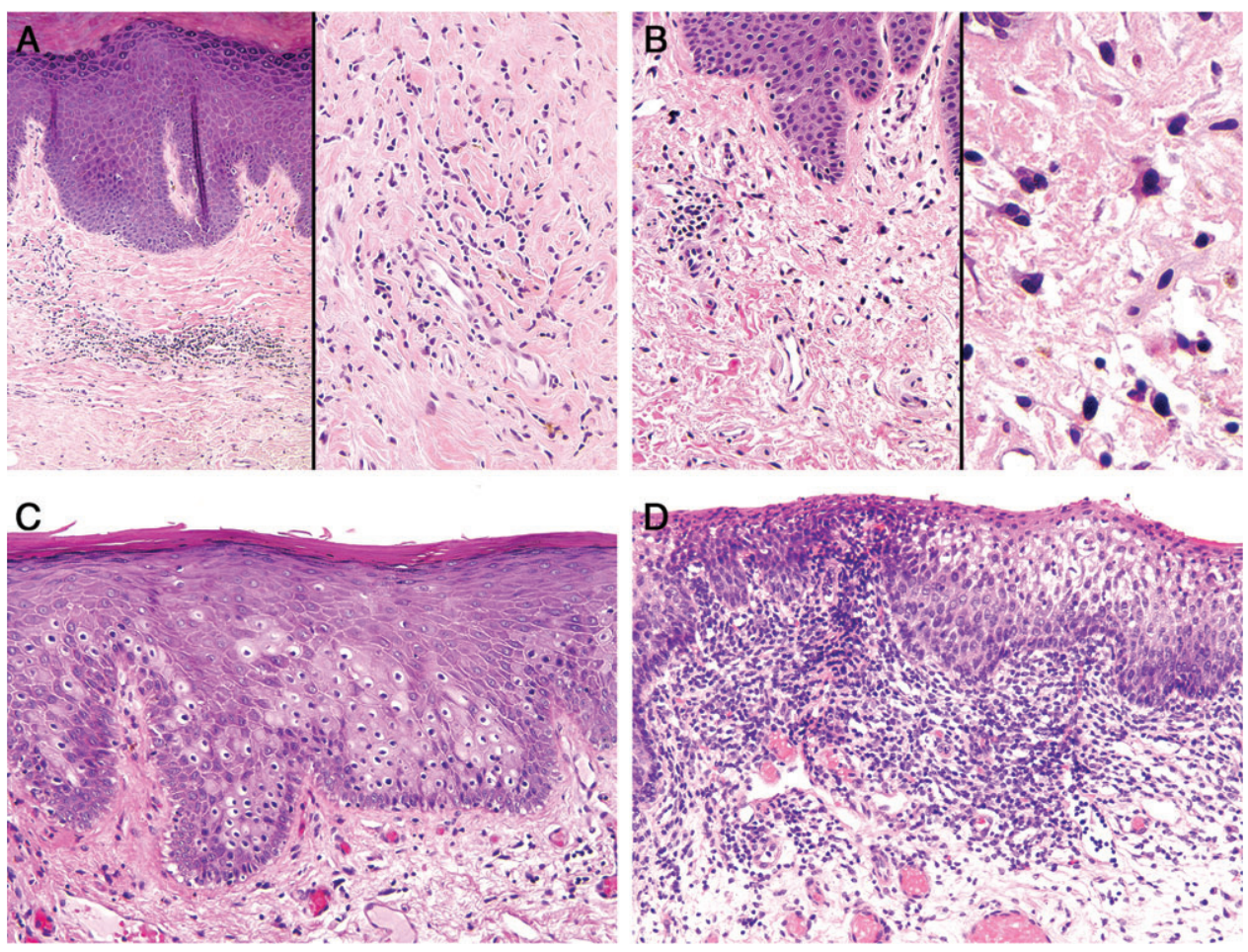

Fig. 2. New histopathologic features observed in vulvar dermatoses. A) Wiry fibrosis with lymphocyte entrapment. A case of lichen sclerosus (LS) with superimposed lichen simplex chronicus (LSC) shows a superficial sclerotic zone underlied by a lymphocytic band (left). At the periphery of the sclerotic zone are thickened collagen fibers with entrapped lymphocytes, giving rise to a distinctive single-filing and branching pattern (right). B) Prominent fibroblasts. A case of LSC shows enlarged stromal fibroblasts, some of which are multinucleated. C) Zones of pale epithelium. Another case of LSC shows discrete clusters of pale keratinocytes at different levels of the epidermis. D) Basal keratinocytic crowding. A case of Zoon vulvitis shows atypical basal keratinocytes with crowding and increased nuclear-to-cytoplasmic ratio. [Hematoxylin-eosin, original magnifications $\times 100$ (A, left), ×200 (A, right), ×200 (B, left), ×800 (B, right), $\times 200(\mathrm{C})$ and $\times 200(\mathrm{D})]$.

next most common diagnoses in our study are LSC and eczematous dermatitis. Interestingly, Zoon vulvitis shows a higher frequency in our series compared to other studies, probably due to heightened awareness and increased detection. As discussed below, we diagnosed Zoon vulvitis by adhering to a constellation of features rather than relying on the sole finding of a dense plasma cell infiltrate.

The rest of the conditions encompass a wide variety of dermatoses which were observed much less frequently. Among these is LP, which showed a slightly lower frequency in our study $(1.6 \%)$ than previously reported by others. ${ }^{4,5}$ This may be due to more successful distinction of LP from other conditions with a band-like infiltrate, such as early inflammatory LS and Zoon vulvitis. Diagnoses of psoriasis, seborrheic dermatitis and sebopsoriasis were made infrequently in the vulvar region. Of note, seborrheic dermatitis and sebopsoriasis represent a clinical spectrum, and their distinction relies on clinical correlation. ${ }^{12}$ The major diagnoses and their histopathologic features are discussed in details below.

\section{Lichen sclerosus (LS)}

The conventional features of LS are well recognized. Completely developed cases are characterized by a subepithelial band of homogenized or sclerotic collagen, with or without an underlying lymphocytic infiltrate. ${ }^{13,14}$ Before homogenization occurs, however, the lymphocytic infiltrate directly interacts with the epidermis/epithelium. The earliest feature of LS, therefore, is that of a lichenoid interface dermatitis. ${ }^{15,16}$ A thickened basement membrane is also considered an early clue. ${ }^{14-19}$ Our retrospective study did not examine the thickness of basement membranes as PAS stain was originally performed in only a small subset of cases.

As lichenoid lymphoid infiltrate is a common feature shared by a number of other dermatoses, early LS continues to be one of the most challenging histopathologic diagnoses. Our study reveals early LS to be most frequently included as part of a differential diagnosis, often considered in conjunction with Zoon vulvitis and LP. We have identified a new histopathologic feature, wiry fibrosis with lymphocyte entrapment 
(Fig. 2A), often observed at the periphery of the subepithelial sclerotic zones in LS. The lymphocytes entrapped between the wiry collagen fibers display a characteristic single-filing or branching pattern which is fairly easy to recognize. In our experience, such alteration in the dermal collagen is similar to that observed in mycosis fungoides. In fact, one study has described 'focally coarse bundles of collagen' in LS (in mostly penile samples) as one of the diagnostic pitfalls simulating early mycosis fungoides. ${ }^{20}$ We believe it is an early feature of LS before a discrete sclerotic zone is formed. Given its significant association with LS, we propose the use of this feature as an indicator of early LS.

Another interesting finding is the presence of eosinophils in almost half of the LS cases. The eosinophils were found in the dermis and the epidermis in 47.9 and $8.5 \%$ of our LS cases, respectively. In comparison, Carlson et al. found dermal and intraepidermal eosinophils in 23.0 and $3.3 \%$ of their vulvar LS cases, respectively. ${ }^{21}$ Our findings suggest a frequent component of hypersensitivity in LS. Interestingly, a previous study has confirmed contact allergies in $47.4 \%$ of patients with LS by patch testing. ${ }^{22}$ This percentage closely mirrors the percentage of eosinophil-containing LS cases in our study. Whether hypersensitivity reaction contributes or predisposes to the development of LS, or merely represents a coincidental finding remains unclear.

\section{Lichen simplex chronicus (LSC)}

Recognizing LSC in vulvar biopsies, whether as a standalone finding or in combination with other conditions, is helpful for the clinician to target therapy that would break the itch-scratch cycle. ${ }^{23}$ Mucosa affected by LSC may become microscopically indistinguishable from skin due to aberrant development of the granular layer and keratinization. Vertically oriented collagen fibers within the papillary dermis, a feature associated with extragenital LSC, has been described as an uncommon feature in vulvar lesions. ${ }^{24}$ Our experience concurs with this observation. Instead of vertical fibrosis, vulvar LSC is more likely to show prominent fibroblasts (Fig. 2B), an observation close to reaching statistical significance. The stromal cells are enlarged and may be multinucleated, and often contain stellate cytoplasm. Such difference in the form of fibrosis is perhaps secondary to the different stromal constituents present in the genitalia.
Our study has also identified zones of pale epithelium (Fig. 2C) as a new histopathologic feature associated with vulvar LSC. It refers to distinct clusters of pale squamous cells within different levels of the epithelium/epidermis. The pale cells contain perinuclear halos and light-staining cytoplasm. Unlike koilocytes, their nuclei remain small and regular, and may be pyknotic. This phenomenon may be identical to 'pagetoid dyskeratosis' previously described in a variety of benign papules. ${ }^{25}$ Although its resemblance to Paget's disease is considered a diagnostic pitfall, the pale cells in pagetoid dyskeratosis may be distinguished from true Paget's cells by their presence of intercellular bridges and lack of intracytoplasmic vacuoles. ${ }^{26}$ Immunohistochemical studies have showed premature keratinization within these pale cells. ${ }^{26,27}$ Although the exact etiology of pagetoid dyskeratosis remains elusive, it is generally believed to represent small clones of keratinocytes that are induced to proliferate under certain circumstances such as chronic friction. ${ }^{25,27}$ This hypothesis correlates well with our finding.

\section{Eczematous (spongiotic) dermatitis}

Included in this category are contact and atopic dermatitides characterized by spongiosis. While a spongiotic pattern is common to a variety of inflammatory conditions, contactants are believed to play an important role and account for most cases of vulvar eczematous dermatitis. ${ }^{28,29}$ From a clinical perspective, the vulvar skin is subject to increased moisture, heat and friction, all of which may weaken its ability to defend against various irritants. ${ }^{28,30}$ Many contactants may also trigger an allergic-type (delayed hypersensitivity) reaction. ${ }^{31}$ Histopathologically, ICD is often difficult to distinguish from ACD. We attempted to subclassify the cases of eczematous dermatitis based on the presence of intraepithelial neutrophils and necrotic keratinocytes in $\mathrm{ICD},{ }^{32,33}$ and eosinophilic spongiosis in ACD. ${ }^{34}$ These changes are well supported by experimental studies. ${ }^{35-37}$ Other cases without these additional findings or showing overlapping features were classified as 'eczema NOS'. In practice, clinical correlation is often needed to distinguish between allergic and irritant contact dermatitides. In our series, most eczematous cases were subclassified as eczema NOS, followed by ACD then ICD. This is contrary to the traditional belief that ICD affects the vulva more frequently than ACD. ${ }^{30}$ As the 


\section{Chan \& Zimarowski}

pathologic changes in ICD are highly dependent on the chemical nature of the specific irritants ${ }^{33}$ and the length of exposure, ${ }^{38}$ it is very likely that some cases of ICD failed to demonstrate necrotic keratinocytes or intraepithelial neutrophils and were therefore classified as 'eczema NOS.' Moreover, patients suffering from ICD are at risk of becoming secondarily sensitized to the various topical agents they use to alleviate their symptoms, ${ }^{31}$ which may have contributed to the higher percentage of ACD observed in our study.

\section{Zoon vulvitis}

Correct diagnosis of Zoon vulvitis requires pathologic confirmation, and carries clinical significance as it may indicate a more refractory course compared to other vulvar dermatoses. To date, large therapeutic trials for Zoon vulvitis are lacking, and a number of treatment modalities have been tried with limited success. ${ }^{39-43}$

The characteristic dense band-like plasma cell infiltrate in Zoon vulvitis is typically accompanied by erosion, intraepithelial neutrophils and hemorrhage/hemosiderin deposition. Other reported features include epithelial atrophy, mild spongiosis, basal vacuolar change, 'lozenge-shaped' keratinocytes, prominent blood vessels and fibrosis. ${ }^{39,44-46}$ Using a constellation of these features, we have identified 15 cases in which Zoon vulvitis is the single best diagnosis, or considered in the differential diagnosis. Zoon vulvitis was not considered in the original pathology diagnoses in eight of these cases. These findings reflect the difficulty in rendering a diagnosis of Zoon vulvitis when only some of its classic features are present. For example, $8-49 \%$ cases of Zoon vulvitis and Zoon balanitis may show only few plasma cells ${ }^{44,47}$ which, when viewed in conjunction with a band-like infiltrate or spongiosis, may be easily confused with early LS or eczema. Its rather inclusive list of histopathologic features may also lead to diagnostic confusion with Behçet disease (erosion/shallow ulceration), psoriasis (neutrophilic exocytosis) and ICD (neutrophilic spongiosis). Familiarization with all features associated with Zoon vulvitis is therefore key to recognition of this condition.

We have observed a new finding, basal keratinocytic crowding (Fig. 2D), in the majority $(86.7 \%)$ of Zoon vulvitis. In contrast to squamous dysplasia, the crowded basal keratinocytes in Zoon vulvitis remain small and uniform, and transition gradually to the adjacent normal keratinocytes without evidence of a discrete clonal process. The background of spongiosis and associated inflammation further support the reactive nature of this finding. The strong association between basal keratinocytic crowding and Zoon vulvitis helps to distinguish Zoon vulvitis from other vulvar dermatoses such as early LS. From our experience, the unusual combination of intraepithelial neutrophils, crowded basal keratinocytes, band-like lymphoplasmacytic infiltrate and hemorrhage is the most useful clue to the diagnosis of Zoon vulvitis.

\section{Lichen planus (LP)}

One persistent diagnostic dilemma is the distinction between LP and early LS. Our study did not identify any new reliable feature of LP, largely due to the relative rarity of LP in our series. In fact, two of our three LP cases also included early LS as a possible differential diagnosis. While it is unrealistic to draw any definitive conclusion from three cases, our findings in LS at least suggest eosinophils and wiry fibrosis with lymphocyte entrapment to be helpful clues in favoring LS over LP. Demonstration of decreased elastic fibers by elastic-van Gieson (EVG) stain and thickened basement membranes by PAS stain, as reported by previous studies, may further support a diagnosis of early LS over LP. ${ }^{19,48}$

\section{Infections}

Although excluded from this study, the infectious cases that were originally misdiagnosed have illustrated a potential diagnostic pitfall. The most commonly overlooked infection in our review was erythrasma caused by $C$. minutissimum. Microscopically, it may present as an 'invisible dermatosis' or mimic chronic eczematous dermatitis. ${ }^{49}$ Upon close inspection, however, clusters of vertically oriented bacterial bacilli are identifiable within the stratum corneum on hematoxylin-eosin staining. ${ }^{50}$ Screening of the stratum corneum at high magnification is therefore prudent to avoid underdiagnosis of erythrasma. The other missed diagnosis was herpetic infection. As the classic cytopathic changes may be focal, careful examination of the adnexal structures and any ulcerated epithelium is needed. Other helpful clues include periadnexal and perineural inflammation and adnexal dyskeratoses. ${ }^{51}$ Lastly, given the prevalence of human papillomavirus in genital skin, caution should also be taken so as not to overlook any concomitant changes of 
condyloma acuminatum or vulvar intraepithelial neoplasia.

The biggest limitation of this study is the lack of diagnostic 'gold standards'. As clinical data were rather limited in most cases, the final diagnoses were based primarily on histopathologic examination. While well-developed changes (such as those in mid- to late-stage LS and LSC) raise little controversy, other entities may exhibit greater variability in their constellation of histopathologic findings and are inevitably prone to diagnostic subjectivity. Despite such subjectivity, consensus was achieved in the majority of cases in which a single best diagnosis was rendered, whereas a limited differential diagnosis was given to the more challenging or ambiguous cases. We also believe that reviewing a large series of cases in a continuous fashion would improve diagnostic accuracy and add value to this type of study.

In conclusion, classification of vulvar dermatoses remains a challenging area in pathology. A number of new and helpful histopathologic clues to their diagnoses have been described in this study. Besides the vulvar dermatoses encountered in this series, other inflammatory conditions such as fixed drug eruption, graft-versus-host disease and immunobullous disorders may also occur on vulvar skin. Therefore, the importance of keeping a broad differential diagnosis in consideration cannot be overstressed. Correct pathologic classification of vulvar dermatoses will facilitate appropriate management of patients suffering from these often chronic and sometimes debilitating conditions.

\section{References}

1. Lynch PJ. 2006 International Society for the Study of Vulvovaginal Disease classification of vulvar dermatoses: a synopsis. J Low Genit Tract Dis 2007; 11: 1.

2. Lynch PJ, Moyal-Barracco M, Bogliatto F, Micheletti L, Scurry J. 2006 ISSVD classification of vulvar dermatoses: pathologic subsets and their clinical correlates. J Reprod Med 2007; 52: 3.

3. Fischer G, Spurrett B, Fischer A. The chronically symptomatic vulva: aetiology and treatment. Br J Obstet Gynaecol 1995; 102: 773 .

4. O'Keefe RJ, Scurry JP, Dennerstein G, Sfameni S, Brenan J. Audit of 114 non-neoplastic vulvar biopsies. Br J Obstet Gynaecol 1995; 102: 780.

5. Bowen AR, Vester A, Marsden L, Florell SR, Sharp H, Summers P. The role of vulvar skin biopsy in the evaluation of chronic vulvar pain. Am J Obstet Gynecol 2008; 199: 467.e1.

6. Leighton PC, Langley FA. A clinicopathological study of vulval dermatoses. J Clin Pathol 1975; 28: 394.

7. Leibowitch M, Neill S, Pelisse M, Moyal-Baracco M. The epithelial changes associated with squamous cell carcinoma of the vulva: a review of the clinical, histological and viral findings in 78 women. $\mathrm{Br}$ J Obstet Gynaecol 1990; 97: 1135.

8. Carli P, Cattaneo A, De Magnis A, Biggeri A, Taddei G, Giannotti B. Squamous cell carcinoma arising in vulval lichen sclerosus: a longitudinal cohort study. Eur J Cancer Prev 1995; 4: 491.

9. Carlson JA, Ambros R, Malfetano J, et al. Vulvar lichen sclerosus and squamous cell carcinoma: a cohort, case control, and investigational study with historical perspective; implications for chronic inflammation and sclerosis in the development of neoplasia. Hum Pathol 1998; 29: 932.

10. Chiesa-Vottero A, Dvoretsky PM, Hart WR. Histopathologic study of thin squamous cell carcinomas and associated cutaneous lesions: a correlative study of 48 tumors in 44 patients with analysis of adjacent vulvar intraepithelial neoplasia types and lichen sclerosus. Am J Surg Pathol 2006; 30: 310.

11. van de Nieuwenhof HP, Bulten J, Hollema $\mathrm{H}$, et al. Differentiated vulvar intraepithelial neoplasia is often found in lesions, previously diagnosed as lichen sclerosus, which have progressed to vulvar squamous cell carcinoma. Mod Pathol 2011; 24: 297.

12. Dessinioti C, Katsambas A. Seborrheic dermatitis: etiology, risk factors, and treatments: facts and controversies. Clin Dermatol 2013; 31: 343.

13. Wallace HJ, Whimster IW. Vulval atrophy and leukoplakia. Br J Dermatol 1951; 63: 241.

14. Hewitt J. Histologic criteria for lichen sclerosus of the vulva. J Reprod Med 1986; 31: 781.

15. Regauer S, Liegl B, Reich O. Early vulvar lichen sclerosus: a histopathological challenge. Histopathology 2005; 47: 340.

16. LeBoit PE. A thickened basement membrane is a clue to ... lichen sclerosus!. Am J Dermatopathol 2000; 22: 457.

17. Slater DN, Wagner BE. Early vulvar lichen sclerosus: a histopathological challenge (correspondence). Histopathology 2007; 50: 388 .

18. Marren P, Dean D, Charnock M, Wojnarowska $F$. The basement membrane zone in lichen sclerosus: an immunohistochemical study. Br J Dermatol 1997; 136 : 508.

19. Fung MA, LeBoit PE. Light microscopic criteria for the diagnosis of early vulvar lichen sclerosus: a comparison with lichen planus. Am J Surg Pathol 1998; 22: 473.

20. Citarella L, Massone C, Kerl H, Cerroni L. Lichen sclerosus with histopathologic features simulating early mycosis fungoides. Am J Dermatopathol 2003; 25: 463.
21. Carlson JA, Lamb P, Malfetano J, Ambros RA, Mihm MC Jr. Clinicopathologic comparison of vulvar and extragenital lichen sclerosus: histologic variants, evolving lesions, and etiology of 141 cases. Mod Pathol 1998; 11: 844

22. Marren $\mathrm{P}$, Wojnarowska F, Powell S. Allergic contact dermatitis and vulvar dermatoses. Br J Dermatol 1992; 126: 52.

23. Lynch PJ. Lichen simplex chronicus (atopic/neurodermatitis) of the anogenital region. Dermatol Ther 2004; 17: 8 .

24. Ackerman AB. Subtle clues to diagnosis by conventional microscopy. Marked compact hyperkeratosis as a sign of persistent rubbing. Am J Dermatopathol 1980; 2: 149.

25. Mehregan AH. Clear epidermal cells, an artifact. J Cutan Pathol 1980; 7: 154.

26. Val-Bernal JF, Garijo MF. Pagetoid dyskeratosis of the prepuce. An incidental histologic finding resembling extramammary Paget's disease. J Cutan Pathol 2000; 27: 387.

27. Tschen JA, McGavran MH, Kettler AH Pagetoid dyskeratosis: a selective keratinocytic response. J Am Acad Dermatol 1988; 19: 891.

28. Farage M, Maibach HI. The vulvar epithelium differs from the skin: implications for cutaneous testing to address topical vulvar exposures. Contact Dermatitis 2004; 51: 201.

29. Schlosser BJ. Contact dermatitis of the vulva. Dermatol Clin 2010; 28: 697.

30. Britz MB, Maibach HI. Human cutaneous vulvar reactivity to irritants. Contact Dermatitis 1979; $5: 375$.

31. Nardelli A, Degreef H, Goossens A. Contact allergic reactions of the vulva: a 14-year review. Dermatitis 2004; 15: 131.

32. Taylor RM. Histopathology of contact dermatitis. Clin Dermatol 1986; 4: 18. 


\section{Chan \& Zimarowski}

33. Willis CM, Stephens CJM, Wilkinson JD. Differential patterns of epidermal leukocyte infiltration in patch test reactions to structurally unrelated chemical irritants. J Invest Dermatol 1993; 101: 364.

34. Wildemore JK, Junkins-Hopkins JM, James WD. Evaluation of the histologic characteristics of patch test confirmed allergic contact dermatitis. J Am Acad Dermatol 2003; 49: 243.

35. Mahmoud G, Lachapelle JM, van Neste D. Histological assessment of skin damage by irritants: its possible use in the evaluation of a 'barrier cream'. Contact Dermatitis 1984; 11: 179.

36. Newby CS, Barr RM, Greaves MW, Mallet AI. Cytokine release and cytotoxicity in human keratinocytes and fibroblasts induced by phenols and sodium dodecyl sulfate. J Invest Dermatol 2000; 115: 292.

37. Varani J, Perone P, Spahlinger DM, et al. Human skin in organ culture and human skin cells (keratinocytes and fibroblasts) in monolayer culture for assessment of chemically induced skin damage. Toxicol Pathol 2007; 35: 693.

38. Nater JP, Hoedemaeker PJ. Histologic differences between irritant and allergic patch test reactions in man. Contact Dermatitis 1976; 2: 247.

39. Kavanagh GM, Burton PA, Kennedy CTC. Vulvitis chronica plasmacellularis (Zoon vulvitis). Br J Dermatol 1993; 129: 92.

40. Gunter J, Golitz L. Topical misoprostol therapy for plasma cell vulvitis: a case series. J Low Genit Tract Dis 2005; 9: 176.

41. Botros SM, Dieterich M, Sand PK, Goldberg RP. Successful treatment of Zoon vulvitis with high potency topical steroid. Int Urogynecol J Pelvic Floor Dysfunct 2006; 17: 178.

42. Frega A, Rech F, French D. Imiquimod treatment of vulvitis circumscripta plasmacellularis. Int J Gynaecol Obstet 2006; 95: 161.

43. Virgilli A, Mantovani L, Lauriola MM, Marzola A, Corazza M. Tacrolimus $0.1 \%$ ointment: is it really effective in plasma cell vulvitis? Report of four cases. Dermatology 2008; 216: 243.

44. Brix WK, Nassau SR, Patterson JW, Cousar JB, Wick MR. Idiopathic lymphoplasmacellular mucositis-dermatitis. J Cutan Pathol 2010; 37: 426 .

45. Souteyrand P, Wong E, MacDonald DM. Zoon balanitis (balanitis circumscripta plasmacellularis). Br J Dermatol 1981; 105: 195.

46. Nedwich JA, Chong KC. Zoon vulvitis. Australas J Dermatol 1987; 28: 11

47. Weyers W, Ende Y, Schalla W, Diaz-Cascajo C. Balanitis of Zoon. A clinicopathologic study of 45 cases. Am J Dermatopathol 2002; 24: 459.

48. Mihara Y, Mihara M, Hagari Y, Shimao S. Lichen sclerosus et atrophicus. A histological, immunohistochemical and electron microscopic study. Arch Dermatol Res 1994; 286: 434.

49. Blaise G, Nikkels AF, Hermanns-Le T, Nikkels-Tassoudji N, Piérard GE. Corynebacterium-associated skin infections. Int J Dermatol 2008; 47: 884.

50. Mattox TF, Rutgers J, Yoshimori RN, Bhatia NN. Nonfluorescent erythrasma of the vulva. Obstet Gynecol 1993; 81: 862.

51. Böer A, Herder N, Blödorn-Schlicht N, Steinkraus V, Falk TM. Herpes incognito most commonly is herpes zoster and its histopathologic pattern is distinctive!. Am J Dermatopathol 2006; 28: 181. 